\title{
MiR-503 inhibits hepatocellular carcinoma cell growth via inhibition of insulin-like growth factor I receptor
}

This article was published in the following Dove Press journal:

OncoTargets and Therapy

I5 June 2016

Number of times this article has been viewed

\author{
Yao Xiao' \\ Qinggang Tian ${ }^{2}$ \\ Jiantai $\mathrm{He}^{\prime}$ \\ Ming Huang' \\ Chao Yang' \\ Liansheng Gong' \\ 'Department of Hepatobiliary \\ and Pancreatic Surgery, Xiangya \\ Hospital, Central South University, \\ Changsha, Hunan, ${ }^{2}$ Department of \\ General Surgery, The Fourth Affiliated \\ Hospital of Baotou Medical College, \\ Baotou, Inner Mongolia, People's \\ Republic of China
}

\begin{abstract}
MicroRNAs (miRs) have been demonstrated to play key roles in the development and progression of hepatocellular carcinoma (HCC). However, the regulatory mechanism of miR-503 in HCC has not been fully uncovered. In this study, we found that miR-503 was significantly downregulated in HCC tissues compared to nontumorous liver tissues. Moreover, lower miR-503 levels were associated with the malignant progression of $\mathrm{HCC}$, and the expression of miR-503 was also decreased in several common HCC cell lines compared to normal human liver cell line THLE-3. Overexpression of miR-503 inhibited proliferation but induced apoptosis of LM3 and HepG2 cells. Bioinformatical analysis and luciferase reporter assay further identified insulin-like growth factor 1 receptor (IGF-1R) as a novel target of miR-503 in 293T cells. Moreover, overexpression of miR-503 led to a significant decrease in the protein levels of IGF-1R, while knockdown of miR-503 enhanced its protein levels in LM3 and HepG2 cells. Besides, overexpression of IGF-1R reversed the effects of miR-503-mediated HCC cell proliferation and apoptosis, indicating that IGF-1R acts as a downstream effector of miR-503 in HCC cells. Furthermore, IGF-1R was found to be significantly upregulated in HCC tissues compared to nontumorous liver tissues. In addition, the mRNA levels of IGF-1R were inversely correlated to the miR-503 levels in the HCC tissues. Thus, we demonstrate that miR-503 inhibits the proliferation and induces the apoptosis of HCC cells, partly at least, by directly targeting IGF-1R, and suggest that IGF-1R may serve as a promising target for the treatment of HCC.
\end{abstract}

Keywords: hepatocellular carcinoma, microRNA-503, insulin-like growth factor 1 receptor, proliferation, apoptosis

\section{Introduction}

Hepatocellular carcinoma (HCC) is one of the most common human cancers worldwide, and the incidence of $\mathrm{HCC}$ has been increasing rapidly in the last decade. ${ }^{1}$ Although significant progresses in the diagnosis and treatment of HCC have been made, HCC still remains to be the third leading cause of cancer-related deaths worldwide. ${ }^{2}$ Thus, investigations on the molecular mechanism underlying the development and progression of HCC are urgently needed for the development of effective therapeutic strategies.

MicroRNAs (miRs) are a class of noncoding RNAs, 18-25 nucleotides in length, that have been demonstrated to negatively mediate the expression of their target genes by inducing mRNA degradation or suppressing protein translation, via directly binding to $3^{\prime}$ untranslated regions (3UTRs) of their target mRNAs. ${ }^{3}$ Moreover, a variety of miRs have been demonstrated to be involved in the development and progression of HCC by negatively mediating the expression of their targets. ${ }^{4}$ Recently, miR-503 was found to act as a tumor suppressor in several cancer types including HCC. ${ }^{5-7}$ Zhou et al found
Correspondence: Liansheng Gong Department of Hepatobiliary and Pancreatic Surgery, Xiangya Hospital, Central South University, Xiangya

Road 87, Changsha, Hunan 410008 ,

People's Republic of China

Tel +86731 89753060

Email gongliansheng8280@I63.com 
that miR-503 induced a G1 arrest and decreased proliferation in HCC HCCLM3 cells and inhibited migration and invasion of HCCLM3 cells. ${ }^{8}$ Besides, miR-503 was also found to inhibit the angiogenesis and growth of $\mathrm{HCC}$ via directly targeting FGF2 and VEGFA. ${ }^{9}$

Insulin-like growth factor 1 receptor (IGF-1R) can bind IGF with a high affinity. Besides, IGF-1R shows tyrosine kinase activity and plays a critical role in transformation events. ${ }^{10}$ Moreover, IGF-1R is highly expressed in most malignant tissues, where it promotes cell survival and inhibits cell apoptosis. ${ }^{10}$ Therefore, IGF-1R has been suggested as a potential target for cancer treatment. ${ }^{11,12}$ Recently, specific miRs have been found to directly target IGF-1R, and thus play suppressive roles in several cancer types. MiR-323-5p inhibits glioma by directly targeting IGF-1R. ${ }^{13}$ Moreover, miR-100, miR-122, and miR-133a have been demonstrated to function as tumor suppressors by targeting IGF-1R in HCC. ${ }^{14-16}$

In the present study, we aimed to investigate the expression of miR-503 in HCC tissues and cell lines. We also performed in vitro study to reveal the role as well as the mechanism of action of miR-503 in HCC cell proliferation and apoptosis, involving IGF-1R. Our data showed that miR-503, downregulated in HCC tissues and cells, was associated with the malignant progression of HCC and could inhibit the proliferation while inducing the apoptosis of HCC cells, partly at least, via directly targeting IGF-1R.

\section{Materials and methods}

\section{Tissue collection}

Our study was approved by the Ethics Committee of Xiangya Hospital of Central South University, Changsha, People's Republic of China. Primary HCC tissues $(n=87)$ and nontumorous liver tissues $(n=8)$ were collected from Xiangya Hospital of Central South University from January 2012 to December 2012. The tumor tissue and nontumor tissue the samples are not taken from the same patient. Among the HCC patients included in the study, 37 were female and 50 were male with the age ranging from 34 to 73 years, with a mean of 51.2 years. The clinicopathologic characteristics, including tumor size, hepatitis B virus infection, node metastasis, vascular invasion, histological grade, clinical stage, and alpha fetal protein (AFP) levels, are summarized in Table 1. All patients received neither radiation therapy nor chemotherapy before surgical resection. All subjects had confirmed pathological diagnosis and were classified according to the World Health Organization (WHO) criteria. Written informed consent was obtained from each participant. Tissues were immediately snap-frozen in liquid nitrogen after surgical removal.
Table I Clinicopathologic characteristics of patients with hepatocellular carcinoma

\begin{tabular}{|c|c|}
\hline Variants & Data \\
\hline \multicolumn{2}{|l|}{ Age (years) } \\
\hline Mean \pm SD & $51.2 \pm 15.3$ \\
\hline \multicolumn{2}{|l|}{ Sex (\%) } \\
\hline Male & $57.5(50 / 87)$ \\
\hline Female & $42.5(37 / 87)$ \\
\hline \multicolumn{2}{|l|}{ Tumor number, \% } \\
\hline Solitary & $85.1(74 / 87)$ \\
\hline Multiple & $14.9(13 / 87)$ \\
\hline \multicolumn{2}{|l|}{ Tumor size $(\mathrm{cm})$} \\
\hline Mean \pm SD & $4.38 \pm 3.54$ \\
\hline \multicolumn{2}{|l|}{ Histologic grade, \% } \\
\hline Well differentiated & $14.9(13 / 87)$ \\
\hline Moderately differentiated & $57.5(50 / 87)$ \\
\hline Poorly differentiated & $27.6(24 / 87)$ \\
\hline \multicolumn{2}{|l|}{ Nodal metastasis, \% } \\
\hline Present & $39.1(34 / 87)$ \\
\hline Absent & $60.9(53 / 87)$ \\
\hline \multicolumn{2}{|l|}{ Vascular invasion, \% } \\
\hline Present & $49.4(43 / 87)$ \\
\hline Absent & $50.6(44 / 87)$ \\
\hline \multicolumn{2}{|l|}{ HBV infection, \% } \\
\hline Present & $90.8(79 / 87)$ \\
\hline Absent & $9.2(8 / 87)$ \\
\hline \multicolumn{2}{|l|}{ Clinical T stage, $\%$} \\
\hline TI & I6.I (I4/87) \\
\hline $\mathrm{T} 2$ & $40.2(35 / 87)$ \\
\hline $\mathrm{T} 3$ & $32.2(28 / 87)$ \\
\hline $\mathrm{T} 4$ & II.5 (I0/87) \\
\hline \multicolumn{2}{|l|}{$\operatorname{AFP}(n g / m L), \%$} \\
\hline$\leq 400$ & $49.4(43 / 87)$ \\
\hline$>400$ & $50.6(44 / 87)$ \\
\hline
\end{tabular}

\section{Real-time PCR assay}

Total RNA of tissues or cells was extracted by using Trizol reagent (Thermo Fisher Scientific, Waltham, MA, USA) according to the manufacturer's protocol. Reverse Transcription Kit (Thermo Fisher Scientific) was used to convert RNA into cDNA according to the manufacturer's protocol. The miR levels were determined using PrimeScript miRNA RT-PCR Kit (Takara, Dalian, People's Republic of China) on ABI 7500 thermocycler (Thermo Fisher Scientific) in accordance with the manufacturer's instructions. U6 gene was used as an internal reference. Expression of mRNA was detected by real-time reverse-transcription polymerase chain reaction (RT-PCR) using the standard SYBR-Green RTPCR Kit (Takara) following the manufacturer's instructions. The specific primer pairs were as follows: IGF-1R, sense: 5'-AGGATATTGGGCTTTACAACCTG-3' and antisense: 5'-GAGGTAACAGAGGTCAGCATTTT-3'; GAPDH as an internal control, sense: 5'-ACAACTTTGGTATCGTGG 
AAGG-3' and antisense: 5'-GCCATCACGCCACAGT TTC- $3^{\prime}$. The reaction condition was $95^{\circ} \mathrm{C}$ for 5 minutes, followed by 40 cycles of denaturation at $95^{\circ} \mathrm{C}$ for 15 seconds and annealing/elongation step at $60^{\circ} \mathrm{C}$ for 30 seconds. The relative expression was analyzed by the $2^{-\Delta \Delta \mathrm{Ct}}$ method.

\section{Cell culture}

Human HCC cell lines (SMCC7721, MHCC97H, LM3, HepG2, and Hep3B), normal liver THLE-3 cells, and 293T cells were obtained from the Cell Bank of Central South University. Cells were cultured in Dulbecco's Modified Eagle's Medium (DMEM, Thermo Fisher Scientific) with $10 \%$ fetal bovine serum (FBS, Thermo Fisher Scientific) at $37^{\circ} \mathrm{C}$ in a humidified incubator containing $5 \% \mathrm{CO}_{2}$.

\section{Transfection}

Cell transfection was conducted using Lipofectamine 2000 (Thermo Fisher Scientific) according to the manufacturer's instructions. In brief, LM3 and HepG2 cells were cultured to $70 \%$ confluence, and resuspended in serum-free DMEM. Scramble miR (miR-NC), miR-503 mimics, miR-503 inhibitor, pc-DNA3.1-IGF-1R plasmid (all obtained from Amspring, Changsha, People's Republic of China), and Lipofectamine 2000 were diluted with serum-free DMEM. The diluted Lipofectamine 2000 was added to the diluted miR or plasmid, incubated for 20 minutes at room temperature, and then added to the cell suspension. After culturing for 6 hours, the medium was replaced with DMEM with $10 \%$ FBS. Then, LM3 and HepG2 cells were cultured for 24 hours before the following assays were performed.

\section{Cell proliferation analysis}

MTT assay was used to determine the cell proliferation. LM3 and HepG2 cells $\left(10^{4}\right)$ were cultured in 96-well plate, and $100 \mu \mathrm{L}$ of fresh serum-free medium with $0.5 \mathrm{~g} / \mathrm{L} \mathrm{MTT}$ (Sigma-Aldrich Co., St Louis, MO, USA) was added to each well. After incubation at $37^{\circ} \mathrm{C}$ for $12,24,48$, and 72 hours, the medium was removed by aspiration and $50 \mu \mathrm{L}$ of dimethyl sulfoxide (Sigma-Aldrich Co.) was added to each well. After incubation at $37^{\circ} \mathrm{C}$ for a further 10 minutes, the absorbance of each sample was measured at $570 \mathrm{~nm}$ using a plate reader (Tecan Infinite M200; Männedorf, Switzerland).

\section{Cell apoptosis assay}

Annexin V-FITC Apoptosis Detection Kit (BD Pharmingen, San Diego, CA, USA) was used to examine the cell apoptosis. LM3 and HepG2 cells $\left(10^{6}\right)$ were washed with cold phosphate-buffered saline (PBS) and resuspended in $200 \mu \mathrm{L}$ of binding buffer with $10 \mu \mathrm{L}$ of fluorescein isothiocyanate (FITC)-labeled annexin V and $5 \mu \mathrm{L}$ of PI, and incubated in the dark for 30 minutes. Then, $300 \mu \mathrm{L}$ binding buffer was added followed by flow cytometry assay (Accuri C6; Beckman Coulter, Brea, CA, USA).

\section{Western blotting}

LM3 and HepG2 cells were solubilized in cold radioimmunoprecipitation assay (RIPA) lysis buffer (Thermo Fisher Scientific) to extract protein, which was separated with $10 \%$ SDS-PAGE (Pierce, Rockford, IL, USA) and transferred onto a polyvinylidene difluoride (PVDF) membrane (Thermo Fisher Scientific). The PVDF membrane was incubated with mouse anti-human IGF-1R monoclonal antibody (1:100, Abcam, Cambridge, UK) or mouse anti-human GAPDH monoclonal antibody $(1: 100$, Abcam $)$ at $4{ }^{\circ} \mathrm{C}$ overnight. After washing with PBS containing 0.6\% Triton-X 100 (PBST) for 15 minutes, the PVDF membrane was incubated with the rabbit anti-mouse secondary antibody (1:1,000, Abcam) at room temperature for 1 hour. Chemiluminescent detection was conducted by using the ECL kit (Pierce). The protein expression was analyzed using Image-Pro plus software 6.0, represented as the density ratio versus GAPDH.

\section{Bioinformatic prediction and dual luciferase reporter assay}

TargetScan (www.targetscan.org) was used to predict the putative target genes of miR-503. The wild type (WT) or the mutant type (MT) of IGF-1R 3'-UTR was constructed by using PCR and Quick-Change Site-Directed Mutagenesis Kit (Stratagene, La Jolla, CA, USA), which was then inserted into the multiple cloning site in the psiCHECK ${ }^{\mathrm{TM}^{2}}$ vector (Promega Corporation, Fitchburg, WI, USA). For luciferase reporter assay, $293 \mathrm{~T}$ cells were cultured to $\sim 60 \%$ confluence in a 24-well plate, and cotransfected with $100 \mathrm{ng}$ of psiCHECK ${ }^{\mathrm{TM}^{2}}$-IGF-1R-3'UTR or psiCHECK ${ }^{\mathrm{TM}^{2}}$-IGF1R-MUT-3'UTR vector, plus $50 \mathrm{nM}$ of miR-503 mimics or scramble miR using Lipofectamine 2000. After incubation for 5 hours, the medium was replaced with DMEM added with 10\% FBS. The dual-luciferase reporter assay system (Promega Corporation) was used to determine the luciferase activity 48 hours after transfection. Renilla luciferase activity was normalized to firefly luciferase activity.

\section{Statistical analysis}

Data are expressed as the mean \pm standard deviation. The statistical correlation of data between groups was analyzed by one-way analysis of variance (ANOVA). The relationship 
between miR-503 expression and HCC clinical features was analyzed using chi-square test. Statistical analysis was performed using SPSS 17.0 (SPSS Inc., Chicago, IL, USA). $P$-value $<0.05$ indicated significant differences.

\section{Results}

\section{MiR-503 is downregulated in HCC and} associated with its malignant progression

To reveal the role of miR-503 in HCC, we first examined the expression of miR-503 in HCC tissues as well as in nontumorous liver tissues. As indicated in Figure 1A, the miR-503 levels were significantly decreased in HCC tissues compared to nontumorous liver tissues. Furthermore, our data showed that lower levels of miR-503 were tightly associated with the malignant progression of $\mathrm{HCC}$, including the histological grade, nodal metastasis, vascular invasion, and clinical stage (Table 2). Besides, no association was found between the miR-503 expression and clinicopathologic characteristics of HCC including age, sex, tumor size and number, HBV infection, and AFP levels (Table 2). Moreover, the expression levels of miR-503 were also decreased in HCC cell lines (SMCC7721, MHCC97H, LM3, HepG2, and Hep3B) compared to normal liver THLE-3 cells (Figure 1B). As LM3 and HepG2 cells showed the most significant decrease in miR-503 expression, we used them in the following experiments (Figure 1B).

\section{MiR-503 suppresses the proliferation while inducing the apoptosis of HCC cells}

To further study the role of miR-503 in HCC, we investigated the effect of miR-503 on HCC cell proliferation

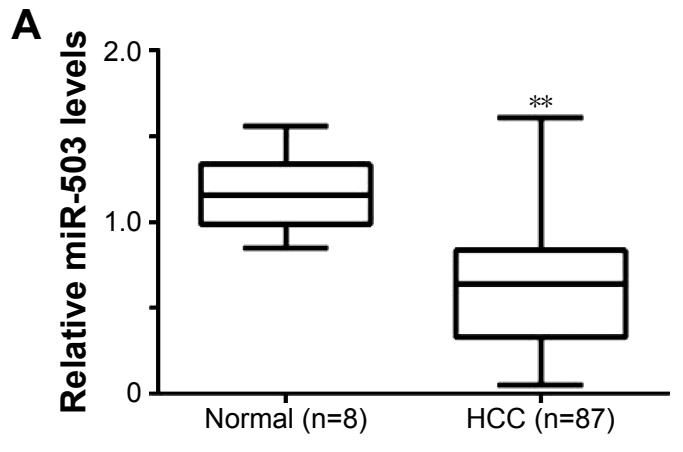

and apoptosis. LM3 and HepG2 cells were transfected with miR-503 mimics to restore its expression levels. Real-time RT-PCR data showed that transfection with miR-503 mimics significantly enhanced the miR-503 levels in LM3 and HepG2 cells, when compared to the control group (Figure 2A). MTT assay was further conducted to examine the cell proliferation in each group. As shown in Figure 2B, overexpression of miR-503 significantly suppressed the proliferation of LM3 and HepG2 cells, suggesting that miR-503 plays an inhibitory role in $\mathrm{HCC}$ cell proliferation. Next, we examined the effect of miR-503 on HCC cell apoptosis. Flow cytometry data indicated that overexpression of miR-503 led to an upregulation of apoptosis in LM3 and HepG2 cells, when compared to the control group (Figure 2C). Accordingly, we demonstrated that miR-503 inhibits HCC cell proliferation and induces cell apoptosis.

\section{IGF-IR is a direct target gene of miR-503 in HCC cells}

Bioinformatical analysis was further conducted to predict the putative target genes of miR-503. TargetScan software predicated that IGF-1R was a direct target gene of miR-503, and this targeting relationship was evolutionally conserved (Figure 3A and B). To confirm their targeting relationship, we generated the luciferase vectors containing WT or MUT of IGF-1R 3'-UTR (Figure 3C and D). Luciferase reporter assay was further conducted in $293 \mathrm{~T}$ cells. As indicated in Figure 3E, the luciferase activity was significantly decreased in $293 \mathrm{~T}$ cells cotransfected with the WT IGF-1R vector and miR-503 mimics, when compared to the control group. However, the luciferase activity was unaltered in $293 \mathrm{~T}$ cells cotransfected with the MUT IGF-1R vector and miR-503

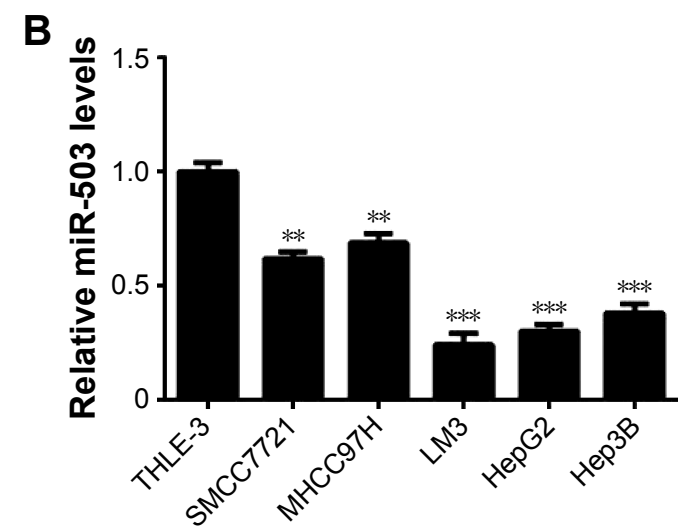

Figure I Expression of miR-503 in HCC tissues and cell lines.

Notes: (A) Real-time RT-PCR was used to determine the relative expression of miR-503 in HCC tissues compared to nontumorous liver tissues (Normal). $* * P<0.01$ vs normal. (B) Real-time RT-PCR was performed to determine the relative expression of miR-503 in human HCC cell lines (SMCC772I, MHCC97H, LM3, HepG2, and Hep3B) compared to normal liver THLE-3 cells. $* * P<0.0$ I vs THLE-3; $* * * P<0.001$ vs THLE-3.

Abbreviations: HCC, hepatocellular carcinoma; RT-PCR, reverse-transcription polymerase chain reaction; miR, microRNA. 
Table 2 Association between miR-503 expression and clinicopathologic characteristics of patients with hepatocellular carcinoma

\begin{tabular}{|c|c|c|c|c|c|}
\hline \multirow[t]{2}{*}{ Variants } & \multicolumn{2}{|c|}{$\begin{array}{l}\text { Low } \\
\text { expression } \\
(n=47) \\
\end{array}$} & \multicolumn{2}{|c|}{$\begin{array}{l}\text { High } \\
\text { expression } \\
(n=40)\end{array}$} & \multirow[t]{2}{*}{$P$-value } \\
\hline & $n$ & $\%$ & $n$ & $\%$ & \\
\hline Age (years) & & & & & 0.976 \\
\hline$\leq 50$ & 21 & 44.7 & 18 & 45 & \\
\hline$>50$ & 26 & 55.3 & 22 & 55 & \\
\hline Sex & & & & & 0.996 \\
\hline Male & 27 & 57.4 & 23 & 57.5 & \\
\hline Female & 20 & 42.6 & 17 & 42.5 & \\
\hline Tumor number & & & & & 0.989 \\
\hline Single & 40 & 85.1 & 34 & 85 & \\
\hline Multiple & 7 & 14.9 & 6 & 15 & \\
\hline Tumor size (cm) & & & & & 0.838 \\
\hline$\leq 5 \mathrm{~cm}$ & 26 & 55.3 & 23 & 57.5 & \\
\hline$>5 \mathrm{~cm}$ & 21 & 44.7 & 17 & 42.5 & \\
\hline Histologic grade & & & & & 0.004 \\
\hline High and moderate & 28 & 59.6 & 35 & 87.5 & \\
\hline Poor & 19 & 40.4 & 5 & 12.5 & \\
\hline Nodal metastasis & & & & & 0.001 \\
\hline Present & 26 & 55.3 & 8 & 20 & \\
\hline Absent & 21 & 44.7 & 32 & 80 & \\
\hline Vascular invasion & & & & & 0.004 \\
\hline Present & 30 & 63.8 & 13 & 32.5 & \\
\hline Absent & 17 & 36.2 & 27 & 67.5 & \\
\hline HBV infection & & & & & 0.894 \\
\hline Present & 42 & 89.4 & 37 & 92.5 & \\
\hline Absent & 5 & 10.6 & 3 & 7.5 & \\
\hline Clinical stage & & & & & 0.005 \\
\hline I-II & 20 & 42.6 & 29 & 72.5 & \\
\hline III-IV & 27 & 57.4 & 11 & 17.5 & \\
\hline $\operatorname{AFP}(\mathrm{ng} / \mathrm{mL})$ & & & & & 0.069 \\
\hline$\leq 400$ & 19 & 40.4 & 24 & 60 & \\
\hline$>400$ & 28 & 59.6 & 16 & 40 & \\
\hline
\end{tabular}

Abbreviations: miR, microRNA; HBV, hepatitis B virus; AFP, alpha fetal protein.

mimics, when compared to the control group (Figure 3E). Accordingly, our data indicated that miR-503 can directly bind to the 3'UTR of IGF-1R.

Further, we investigated the effect of miR-503 on the protein expression of IGF-1R in HCC cells. LM3 and HepG2 cells were transfected with miR-503 mimics and miR-503 inhibitor, respectively. Our data demonstrated that transfection with miR-503 mimics enhanced the miR-503 levels, while transfection with miR-503 inhibitor resulted in a decrease in the miR-503 levels in HCC cells (Figure 3F). We then conducted Western blot to examine the protein levels of IGF-1R in each group. As demonstrated in Figure 3G, overexpression of miR-503 resulted in decreased protein levels of IGF-1R, while knockdown of miR-503 enhanced the protein expression of IGF-1R in HCC cells. Based on the aforementioned data, we demonstrated that miR-503 negatively mediates the protein expression of IGF-1R via directly binding to its $3^{\prime} \mathrm{UTR}$ in HCC cells.

\section{Overexpression of IGF-IR reversed miR- 503-mediated proliferation and apoptosis of HCC cells}

As IGF-1R was confirmed to be a direct target of miR-503, and it has been implicated in the development of HCC, we then studied whether it was involved in miR-503-induced HCC cell proliferation and apoptosis. MiR-503-overexpressing HCC cells were further transfected with IGF-1R plasmid to restore its expression. After transfection, Western blot was conducted to examine the protein levels of IGF-1R. As indicated in Figure 4A, the protein expression of IGF-1R was significantly higher in the miR-503+IGF-1R group, when compared to the miR-503 group. After that, MTT assay was performed to determine the cell proliferation. Our data showed that the proliferating capacities of LM3 and $\mathrm{HepG} 2$ cells were significantly higher in the miR-503+IGF-1R group compared to the miR-503 group (Figure 4B), indicating that restoration of IGF-1R rescued the suppressive effects of miR-503 overexpression on HCC cell proliferation. Then, we examined the cell apoptotic levels in each group, and found that the apoptosis of HCC cells was lower in the miR-503+IGF-1R group compared to the miR-503 group (Figure 4C), indicating that overexpression of IGF-1R rescued miR-503-induced $\mathrm{HCC}$ cell apoptosis. Based on these aforementioned data, we suggest that IGF-1R is involved in the miR-503-mediated proliferation and apoptosis of HCC cells.

\section{IGF-IR is highly upregulated in HCC and inversely correlated to the miR-503 levels}

To further confirm their relationship in HCC tissues, we examined the mRNA levels of IGF-1R in HCC tissues and nontumorous liver tissues. Real-time RT-PCR data indicated that the mRNA levels of IGF-1R were significantly increased in HCC tissues compared to nontumorous liver tissues (Figure 5A). Furthermore, we found that the mRNA levels of IGF-1R were inversely correlated with the miR-503 levels in HCC tissues (Figure 5B). Accordingly, we suggest that downregulation of miR-503 may contribute to the upregulation of IGF-1R, which promotes the survival and proliferation of HCC cells.

\section{Discussion}

In the present study, our data showed that miR-503 was significantly downregulated in HCC tissues and cell lines, 
A

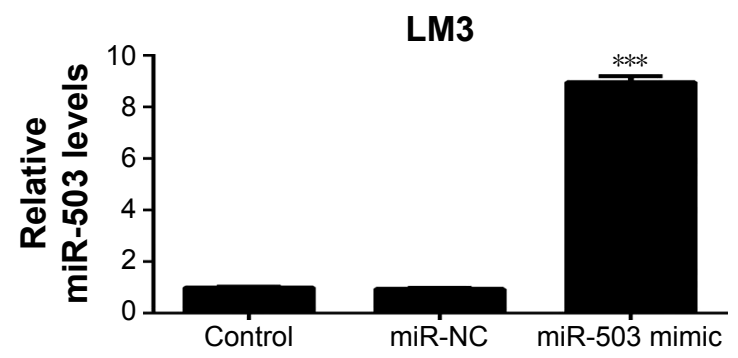

B

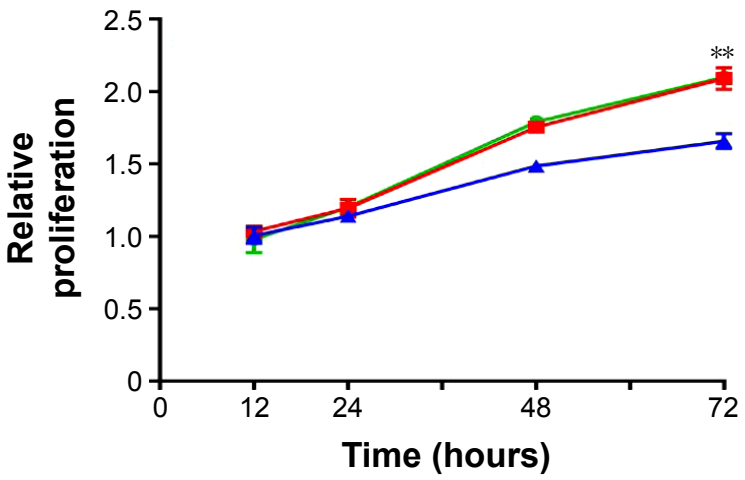

HepG2
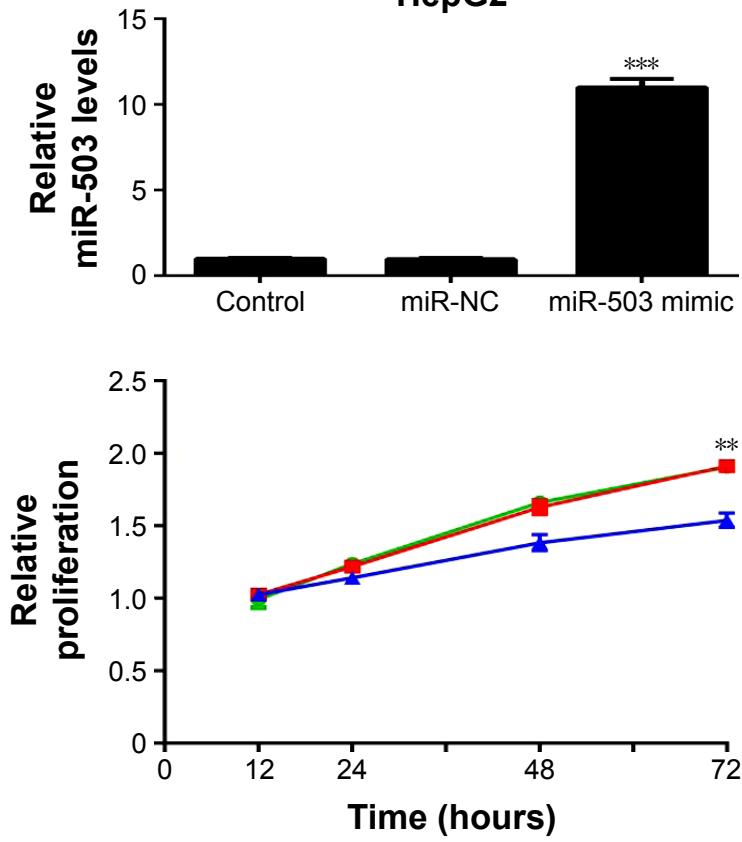

C $\rightarrow$ Control $\rightarrow$ miR-NC $\neq$ miR-503 mimic

Control

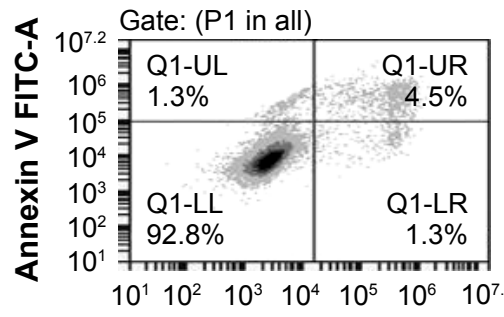

PI

Control

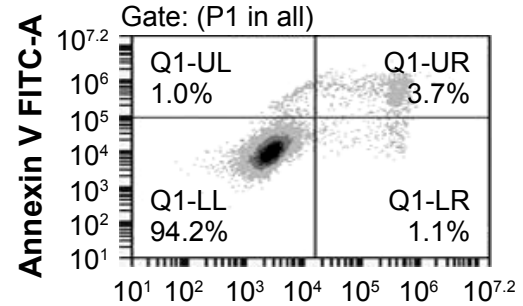

PI
miR-NC

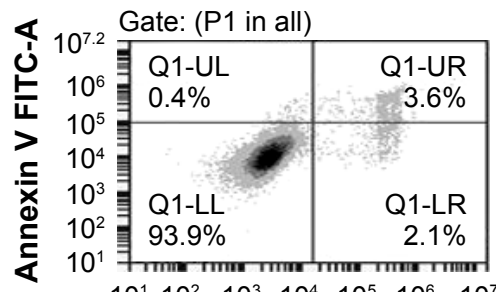

PI

miR-NC

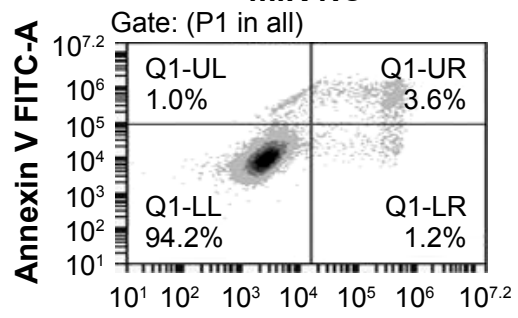

PI
miR-503 mimic

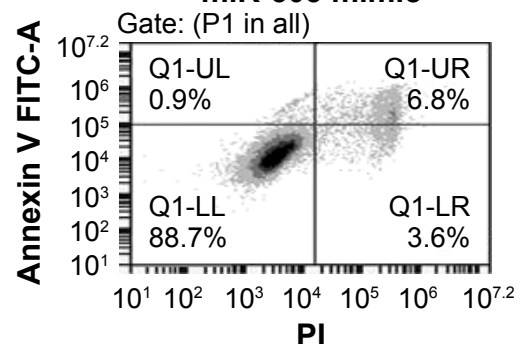

miR-503 mimic

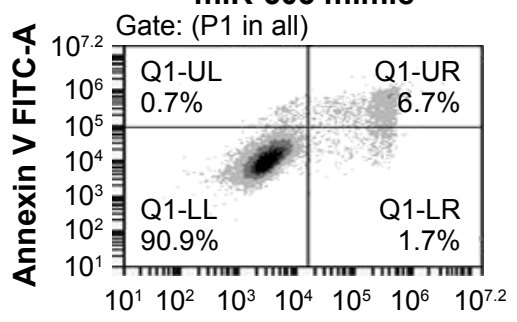

PI
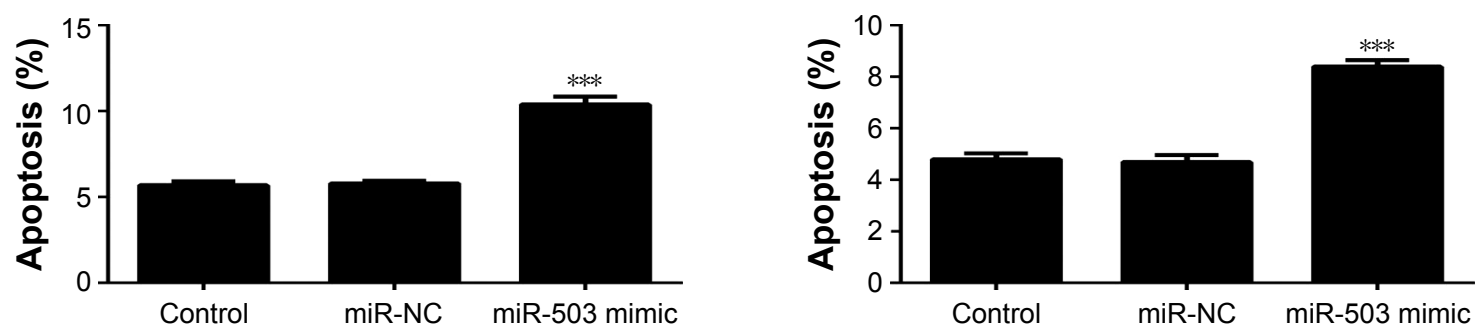

Figure 2 Upregulation of miR-503 inhibits cell growth and induces apoptosis in LM3 and HepG2 cells.

Notes: (A) Real-time RT-PCR was performed to determine the relative expression of miR-503 in LM3 and HepG2 cells transfected with scramble miR (miR-NC) and miR-503 mimic, respectively. (B) MTT assay and (C) flow cytometry were conducted to examine the cell proliferation and apoptosis, respectively. Nontransfected cells were used as control. $* * P<0.01$. $* * * P<0.001$.

Abbreviations: RT-PCR, reverse-transcription polymerase chain reaction; miR, microRNA; NC, negative control; PI, pethidium bromide; UL, upper left; UR, upper right; $\mathrm{LL}$, lower left; LR, lower right. 
A

\begin{tabular}{|c|c|c|c|c|c|c|c|}
\hline & $\begin{array}{c}\text { Predicted consequential pairing of } \\
\text { target region (top) and miRNA (bottom) }\end{array}$ & $\begin{array}{l}\text { Site } \\
\text { type }\end{array}$ & $\begin{array}{c}\text { Context++ } \\
\text { score }\end{array}$ & $\begin{array}{l}\text { Context++ score } \\
\text { percentile }\end{array}$ & $\begin{array}{c}\text { Weighted } \\
\text { context++ score }\end{array}$ & $\begin{array}{c}\text { Conserved branch } \\
\text { length }\end{array}$ & PCT \\
\hline $\begin{array}{l}\text { Position 1269-1275 of IGF-1R 3'UTR } \\
\text { hsa-miR-503-5p }\end{array}$ & 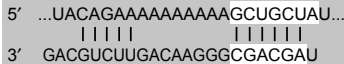 & $\begin{array}{c}7 \mathrm{mer}- \\
\mathrm{A} 1\end{array}$ & -0.29 & 98 & -0.28 & 4.367 & 0.78 \\
\hline
\end{tabular}

B

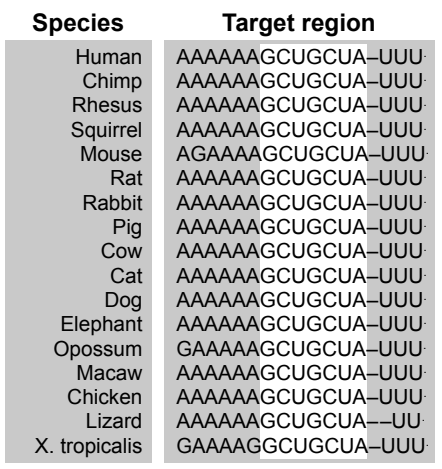

D

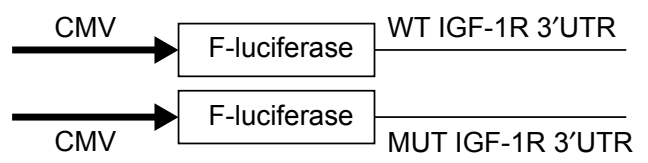

$\mathbf{F}$

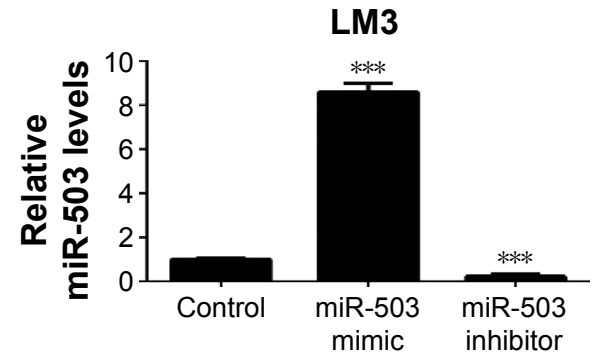

G

\section{LM3}

Control miR-503 miR-503

mimic inhibitor

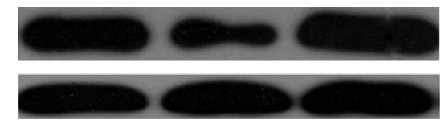

IGF-1R

GAPDH

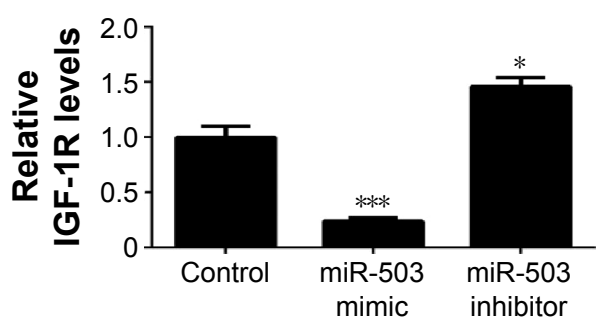

C

\author{
WT IGF-1R 3'UTR $\quad 5^{\prime}$-...AAAAGCUGCUA...-3' \\ |||||| $\mid$ \\ miR-503 3'-...AGGGCGACGAU...-5' \\ | | | | \\ MUT IGF-1R 3'UTR $\quad 5^{\prime}-\ldots$...AAAAGCAAAUA...-3'
}

E

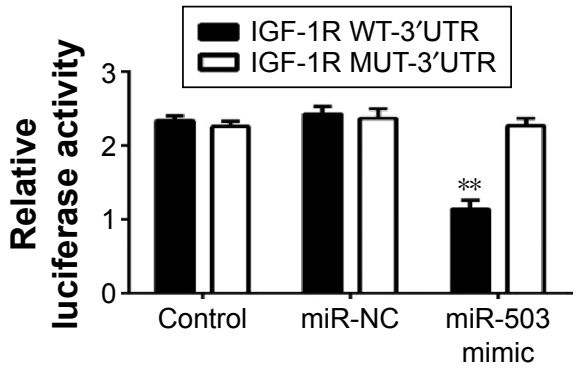

HepG2

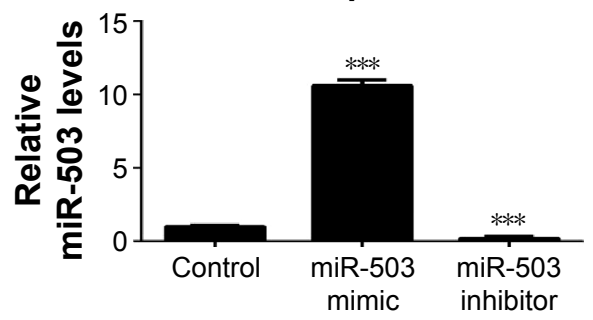

HepG2

Control miR-503 miR-503

mimic inhibitor
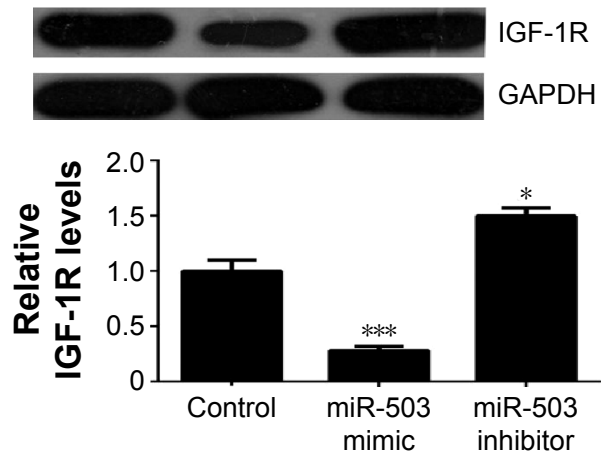

Figure 3 miR-503 directly targets IGF-IR.

Notes: (A, B) TargetScan software predicted that IGF-IR was a target of miR-503, and this targeting relationship was evolutionally conserved. (C) The seed sequences of miR-503 in the wild type (WT) or mutant type (MUT) of IGF-IR 3'-UTR are indicated. (D) The WT or MUT IGF-IR 3'UTR were cloned into luciferase reporter vector. (E) The luciferase activity was significantly decreased in 293T cells cotransfected with the WT IGF-IR vector and miR-503 mimic, but unaltered in 293T cells cotransfected with the MUT IGF-IR vector and miR-503 mimics, compared to the control group. Control, 293T cells transfected with only WT IGF-IR or MUT IGF-IR vectors; NC, 293T cells cotransfected with WT IGF-IR or MUT IGF-IR vector and scramble miR. (F) Real-time RT-PCR was performed to determine the miR-503 levels in LM3 and HepG2 cells transfected with miR-503 mimic and miR-503 inhibitor, and (G) the protein levels of IGF-IR were then examined using Western blot assay. Nontransfected cells were used as control. $* * P<0.01$ vs control. $* * * P<0.001$.

Abbreviations: RT-PCR, reverse-transcription polymerase chain reaction; miR, microRNA; IGF-IR, insulin-like growth factor I receptor; UTR, untranslated region; CMV, cytomegalovirus; PCT, probability of conserved targeting. 
A
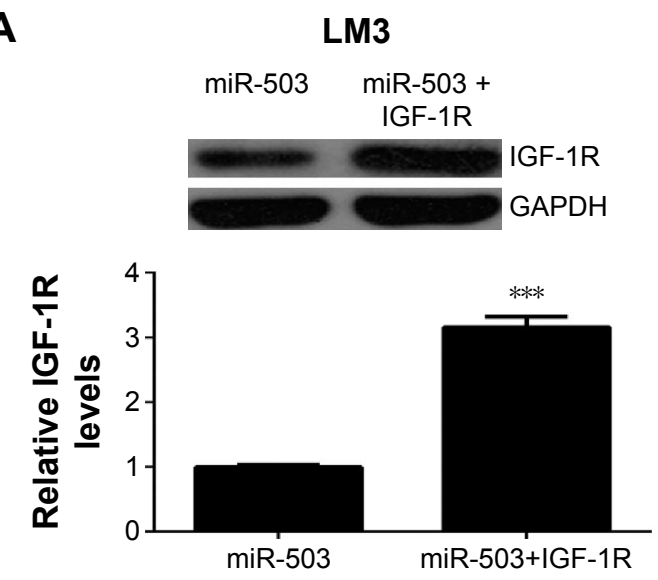

B

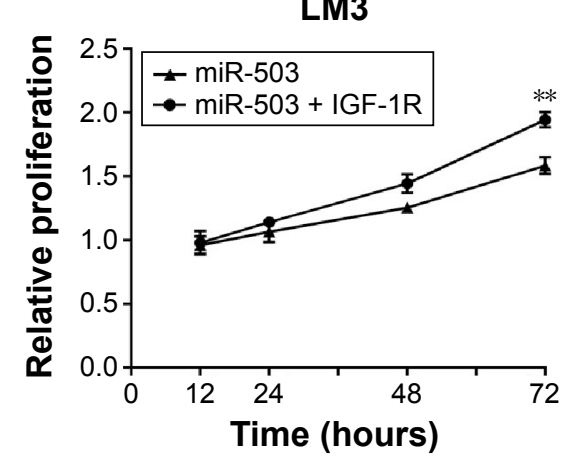

C

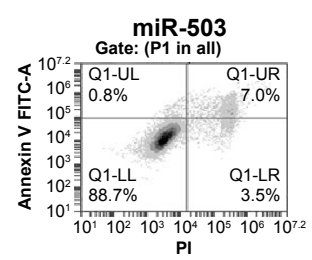

LM3
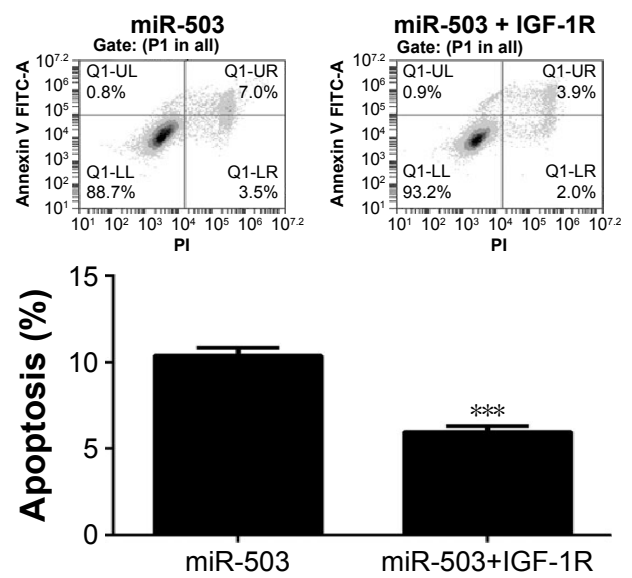

\section{HepG2}

miR-503 miR-503 +

IGF-1R
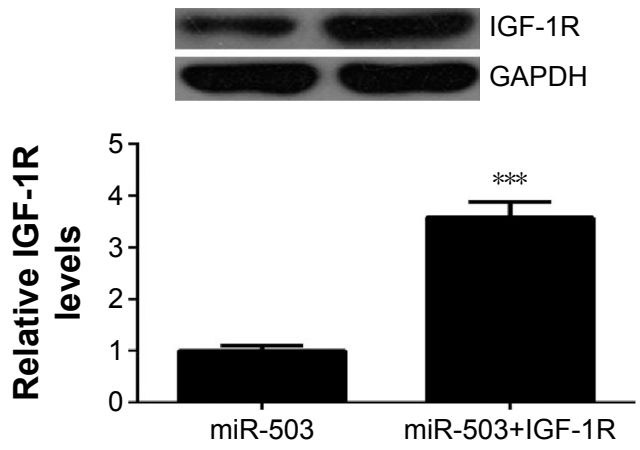

HepG2

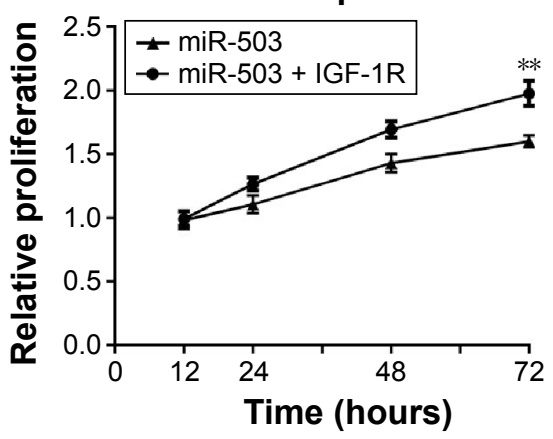

HepG2
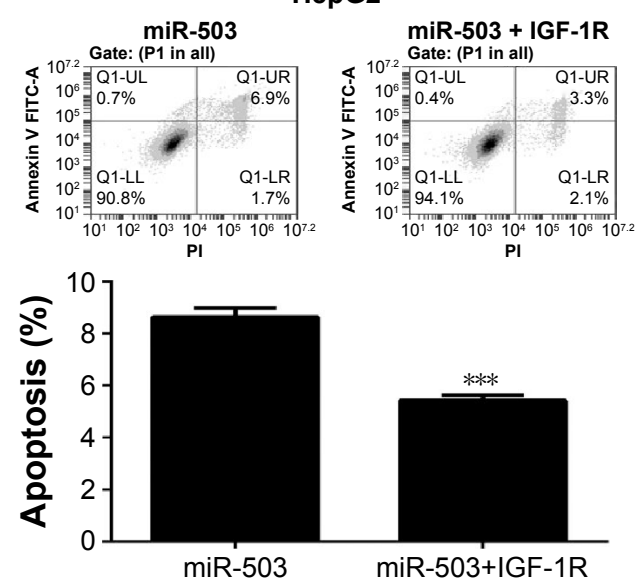

Figure 4 Overexpression of IGF-IR reverses miR-503-mediated suppressive effects on LM3 and HepG2 cells.

Notes: LM3 and HepG2 cells were transfected with miR-503 mimic with or without IGF-IR plasmid. (A) The protein levels of IGF-IR were then examined using Western blot assay. (B) MTT assay and (C) flow cytometry were conducted to examine the cell proliferation and apoptosis, respectively. $* * P<0.01$ vs miR-503. $* * * P<0.00 \mathrm{I}$.

Abbreviations: miR, microRNA; IGF-IR, insulin-like growth factor I receptor; PI, pethidium bromide; UL, upper left; UR, upper right; LL, lower left; LR, lower right.

and lower miR-503 levels were strongly associated with higher malignance of HCC. In vitro investigation showed that overexpression of miR-503 suppressed the proliferation and induced the apoptosis of HCC cells. IGF-1R was further identified as a direct target gene of miR-503 and acted as a downstream effecter in miR-503-mediated HCC cell proliferation and apoptosis. In addition, IGF-1R was significantly upregulated in $\mathrm{HCC}$ tissues and inversely correlated with the miR-503 levels.
Recently, miR-503 has been reported to generally act as a tumor suppressor in different cancer types. ${ }^{17-20}$ For instance, Guo et al found that miR-503 inhibited the proliferation and metastasis of prostate cancer cells by directly targeting RNF31. ${ }^{21}$ Liu et al reported that decreased miR-503 levels were associated with advanced mythological features and poor prognosis in patients with non-small-cell lung cancer. ${ }^{6}$ Long et al demonstrated that miR-503 inhibited cell proliferation of human breast cancer cells by targeting CCND1. ${ }^{22}$ 
A

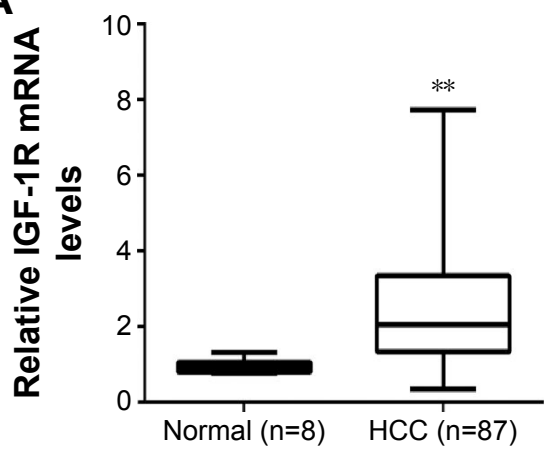

B

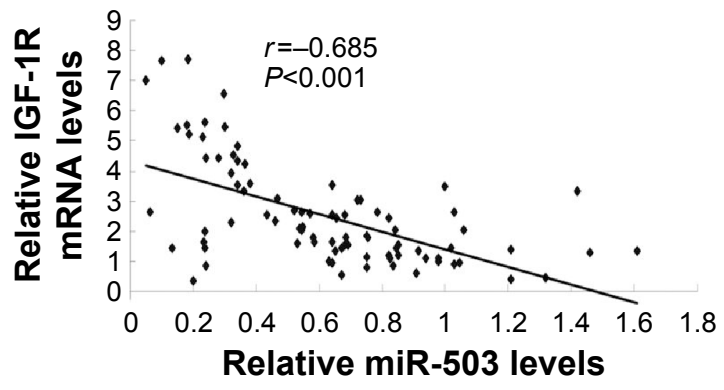

Figure 5 IGF-IR is inversely correlated with miR-503 in HCC tissues.

Notes: (A) Real-time RT-PCR was used to determine the mRNA expression of IGF-IR in HCC tissues compared to nontumorous liver tissues (Normal). ** $<0.0$ I vs normal. (B) The correlation between the mRNA levels of IGF-IR and miR-503 was determined in HCC tissues.

Abbreviations: HCC, hepatocellular carcinoma; RT-PCR, reverse-transcription polymerase chain reaction; miR, microRNA; IGF-IR, insulin-like growth factor I receptor.

However, it was also found to be upregulated in esophageal cancer, and high miR-503 levels were significantly associated with large tumor size and poorer prognosis. ${ }^{23}$ Besides, it was also found to contribute to the malignant transformation of colon adenoma to adenocarcinomas via targeting the tumor suppressor FBXW7. ${ }^{24}$ Accordingly, its exact role is tumor-specific. In the present study, we found that miR-503 was significantly downregulated in HCC tissues and cell lines, and lower miR-503 levels were associated with higher malignance of HCC. Our data are consistent with several other studies. Zhou et al also reported that the expression of miR-503 was repressed in HCC cells and primary tumors due to a potential epigenetic mechanism. ${ }^{25}$ Xiao et al reported that low miR-503 levels were associated with enhanced malignant potential, such as histologic grade, tumor-node-metastasis stage, and AFP level, as well as worse overall survival in HCC patients. ${ }^{26}$ Therefore, our data and those reported by others indicate that downregulation of miR-503 is involved in the development and progression of HCC.

Our data further showed that restoration of miR-503 significantly inhibited the proliferation and induced the apoptosis of HCC cells. Xiao et al also reported that miR-503 suppressed the proliferation of $\mathrm{HCC}$ cells, consistent with ours, and they revealed that miR-503 could induce a G1 phase arrest through targeting two cell cycle-related molecules, cyclin D3 and E2F3. ${ }^{26}$ Besides, Zhou et al reported that overexpression of miR-503 not only inhibited the proliferation but also the metastasis of HCC in vivo. ${ }^{27}$ They also confirmed the negative correlation between miR-503 expression and metastatic potential of HCC in cell lines and in clinical HCC tissues. ${ }^{27}$ Furthermore, they identified ARHGEF19 as a direct target gene of miR-503, and found that ARHGEF19 rescued the suppressive effects of miR-503 on the proliferation and metastasis of HCC cells. ${ }^{27}$ Besides, PRMT1 was also found to be a direct target gene of
miR-503 in HCC. Li et al reported that miR-503 significantly inhibited the invasion, migration, and epithelial-mesenchymal transition of HCC cells via directly targeting PRMT1.?

As one miR has various targets, other important targets of miR-503 may also exist in HCC. Bioinformatic prediction and luciferase reporter assay identified IGF-1R as a direct target gene of miR-503, and the protein levels of IGF-1R were negatively mediated by miR-503 in HCC cells. IGF-1R functions as a key oncogene in the development and maintenance of cancer. ${ }^{28}$ It was reported that IGF-1R could signal in the absence of the ligand and kinase activity, and utilize the components of the GPCR system. ${ }^{28}$ Moreover, IGF-1R antibodies have recently been found to serve as important agents for cancer therapy. ${ }^{10}$ Yue et al generated a murine anti-IGF-1R antibody $4 \mathrm{~F} 2$, and found that treatment with 4F2 decreased cell proliferation and promoted apoptosis of HCC cells..$^{29}$ In our study, we found that miR-503 repressed the protein expression of IGF-1R, and inhibited HCC cell proliferation and induced the cell apoptosis. Moreover, we found that overexpression of IGF-1R reversed the effect of miR-503 on HCC cell proliferation and apoptosis, indicating that IGF-1R is involved in miR-503-meditated proliferation and apoptosis of HCC cells. In addition, IGF-1R was found to be highly expressed in $\mathrm{HCC}$ tissues and inversely correlated to the miR-503 levels, which further suggests that the upregulation of IGF-1R may be due to the downregulation of miR-503 in HCC.

\section{Conclusion}

In summary, the present study demonstrates that miR-503, downregulated in HCC, can inhibit the proliferation but induce the apoptosis of $\mathrm{HCC}$ cells through direct inhibition of IGF-1R expression. Therefore, we suggest that miR-503 may serve as a potential therapeutic agent and IGF-1R as a therapeutic target for HCC. 


\section{Disclosure}

The authors report no conflicts of interest in this work.

\section{References}

1. Torre LA, Bray F, Siegel RL, et al. Global cancer statistics, 2012. CA Cancer J Clin. 2015;65(2):87-108.

2. Siegel RL, Miller KD, Jemal A. Cancer statistics, 2015. CA Cancer J Clin. 2015;65(1):5-29.

3. Treiber T, Treiber N, Meister G. Regulation of microRNA biogenesis and function. Thromb Haemost. 2012;107(4):605-610.

4. Hung $\mathrm{CH}$, Chiu YC, Chen $\mathrm{CH}, \mathrm{Hu}$ TH. MicroRNAs in hepatocellular carcinoma: carcinogenesis, progression, and therapeutic target. Biomed Res Int. 2014;2014:486407.

5. Bassampour SA, Abdi R, Bahador R, et al. Downregulation of miR133b/miR-503 acts as efficient prognostic and diagnostic factors in patients with osteosarcoma and these predictor biomarkers are correlated with overall survival. Tumour Biol. Epub 2015 Aug 15.

6. Liu L, Qu W, Zhong Z. Down-regulation of miR-503 expression predicate advanced mythological features and poor prognosis in patients with NSCLC. Int J Clin Exp Pathol. 2015;8(5):5609-5613.

7. Li B, Liu L, Li X, Wu L. miR-503 suppresses metastasis of hepatocellular carcinoma cell by targeting PRMT1. Biochem Biophys Res Commun. 2015;464(4):982-987.

8. Zhou J, Wang W. Analysis of microRNA expression profiling identifies microRNA-503 regulates metastatic function in hepatocellular cancer cell. J Surg Oncol. 2011;104(3):278-283.

9. Zhou B, Ma R, Si W, et al. MicroRNA-503 targets FGF2 and VEGFA and inhibits tumor angiogenesis and growth. Cancer Lett. 2013;333(2): 159-169.

10. King H, Aleksic T, Haluska P, Macaulay VM. Can we unlock the potential of IGF-1R inhibition in cancer therapy? Cancer Treat Rev. 2014;40(9):1096-1105.

11. Singh $P$, Alex JM, Bast F. Insulin receptor (IR) and insulin-like growth factor receptor 1 (IGF-1R) signaling systems: novel treatment strategies for cancer. Med Oncol. 2014;31(1):805.

12. Chen HX, Sharon E. IGF-1R as an anti-cancer target - trials and tribulations. Chin J Cancer. 2013;32(5):242-252.

13. Lian HW, Zhou Y, Jian ZH, Liu RZ. MiR-323-5p acts as a tumor suppressor by targeting the insulin-like growth factor 1 receptor in human glioma cells. Asian Pac J Cancer Prev. 2014;15(23):10181-10185.

14. Zhang W, Liu K, Liu S, et al. MicroRNA-133a functions as a tumor suppressor by targeting IGF-1R in hepatocellular carcinoma. Tumour Biol. 2015;36(12):9779-9788.

15. Ge YY, Shi Q, Zheng ZY, et al. MicroRNA-100 promotes the autophagy of hepatocellular carcinoma cells by inhibiting the expression of mTOR and IGF-1R. Oncotarget. 2014;5(15):6218-6228.
16. Zeng C, Wang R, Li D, et al. A novel GSK-3 beta-C/EBP alpha-miR122-insulin-like growth factor 1 receptor regulatory circuitry in human hepatocellular carcinoma. Hepatology. 2010;52(5):1702-1712.

17. Yang Y, Liu L, Zhang Y, et al. MiR-503 targets PI3K p 85 and IKK-beta and suppresses progression of non-small cell lung cancer. Int J Cancer. 2014;135(7):1531-1542.

18. Wang T, Ge G, Ding Y, et al. MiR-503 regulates cisplatin resistance of human gastric cancer cell lines by targeting IGF1R and BCL2. Chin Med J (Engl). 2014;127(12):2357-2362.

19. Peng Y, Liu YM, Li LC, Wang LL, Wu XL. microRNA-503 inhibits gastric cancer cell growth and epithelial-to-mesenchymal transition. Oncol Lett. 2014; 7(4):1233-1238.

20. Chong Y, Zhang J, Guo X, et al. MicroRNA-503 acts as a tumor suppressor in osteosarcoma by targeting L1CAM. PLoS One. 2014;9(12): e114585.

21. Guo J, Liu X, Wang M. miR-503 suppresses tumor cell proliferation and metastasis by directly targeting RNF31 in prostate cancer. Biochem Biophys Res Commun. 2015;464(4):1302-1308.

22. Long J, Ou C, Xia H, Zhu Y, Liu D. MiR-503 inhibited cell proliferation of human breast cancer cells by suppressing CCND1 expression. Tumour Biol. 2015;36(11):8697-8702.

23. Ide S, Toiyama Y, Shimura T, et al. MicroRNA-503 promotes tumor progression and acts as a novel biomarker for prognosis in oesophageal cancer. Anticancer Res. 2015;35(3):1447-1451.

24. Li L, Sarver AL, Khatri R, et al. Sequential expression of miR-182 and miR-503 cooperatively targets FBXW7, contributing to the malignant transformation of colon adenoma to adenocarcinoma. $J$ Pathol. 2014;234(4):488-501.

25. Zhou B, Ma R, Si W, et al. MicroRNA-503 targets FGF2 and VEGFA and inhibits tumor angiogenesis and growth. Cancer Lett. 2013;333(2): 159-169.

26. Xiao F, Zhang W, Chen L, et al. MicroRNA-503 inhibits the G1/S transition by downregulating cyclin D3 and E2F3 in hepatocellular carcinoma. J Transl Med. 2013;11:195.

27. Zhou J, Tao Y, Peng C, Gu P, Wang W. miR-503 regulates metastatic function through Rho guanine nucleotide exchanger factor 19 in hepatocellular carcinoma. J Surg Res. 2014;188(1):129-136.

28. Crudden C, Girnita A, Girnita L. Targeting the IGF-1R: The Tale of the Tortoise and the Hare. Front Endocrinol (Lausanne). 2015;6:64.

29. Yue L, Wang Y, Wang H, et al. Inhibition of hepatocellular carcinoma cell growth by an anti-insulin-like growth factor-I receptor monoclonal antibody. Oncol Rep. 2012;28(4):1453-1460.
OncoTargets and Therapy

\section{Publish your work in this journal}

OncoTargets and Therapy is an international, peer-reviewed, open access journal focusing on the pathological basis of all cancers, potential targets for therapy and treatment protocols employed to improve the management of cancer patients. The journal also focuses on the impact of management programs and new therapeutic agents and protocols on

\section{Dovepress}

patient perspectives such as quality of life, adherence and satisfaction. The manuscript management system is completely online and includes a very quick and fair peer-review system, which is all easy to use. Visit http://www.dovepress.com/testimonials.php to read real quotes from published authors. 